\title{
Modulation of Mast Cell Function by Amino Acids In vitro: A Potential Mechanism of Immunonutrition for Wound Healing
}

\author{
Masako Kawamoto and Kayo Masuko* \\ Graduate School of Nutritional Science, Sagami Women's University, Kanagawa, Japan
}

Received: December 14, 2013; Accepted: December 24, 2013; Published: December 26, 2013

*Corresponding author: Kayo Masuko, Graduate School of Nutritional Science, Sagami Women's University, Kanagawa, Japan, Tel: 81-042-7135077; Fax: 81-042-713-5077; E-mail: k_msk@mac.com

\begin{abstract}
Mast cells release important chemical mediators, such as histamine and interleukin (IL)-13, for the regulation of allergic reactions and inflammation. Recently, mast cell activation has been implicated in wound healing. Glutamine (Gln) and Arginine (Arg) are used as "immune nutrients" in severe infections and chronic wounds in malnourished patients, but the potential effect of these amino acids on mast cell activation is unclear. We evaluated the effect of Gln and / or Arg in culture on mast cell function.
\end{abstract}

The murine mast cell line P815 was cultured in RPMI-1640 medium under different Gln and/or Arg concentrations and IL-6 stimulation. Histamine and IL-13 release into the supernatant was measured using enzyme-linked immunoassays.

As a result, the amino acid concentrations affected cellular viability. Cells cultured in higher Gln concentrations produced a greater histamine response upon IL-6 stimulation while the histamine response in cells cultured in a high Arg concentration was suppressed. IL-13 production in the Gln containing medium was not significantly altered by IL-6 stimulation.

In conclusion, in an inflammatory milieu, Gln and/or Arg concentrations in situ may regulate histamine and IL-13 release from mast cells. The appropriate use of functional amino acids as immune nutrients may suppress inflammation and aid in wound healing.

Keywords: Glutamine; Arginine; Mast cells; Histamine; IL-13

\section{Introduction}

Mast cells, which are derived from pluripotent stem cells, reside in the submucosa or connective tissue in mammals and play important roles in the innate immune and inflammatory responses, including those involved in allergic reactions. Mast cells contain a number of granules that are rich in chemical mediators, such as histamine and heparin. Mast cells are stimulated to release these mediators crosslinked on the cell surface by immunoglobulin E (IgE), when it has been induced by an antigen. On the other hand, non-IgE-dependent pathways for mast cell activation are also present [1-3]. For example, pro- inflammatory cytokines such as interleukin (IL)-6 can induce the release of histamine from mast cells, at least in vitro [4].

Accumulating evidence suggests a potential enhancing effect of mast cells in the process of wound healing [5-7]. In animal models, mice that lack mast cells demonstrate delayed wound healing compared to their normal littermates [8]. One potential mechanism by which mast cells are involved in wound healing is mast cell-derived histamine, as histamine might promote fibroblast migration [9]. An additional role of mast cells in wound healing might be related to IL-13, which is generally accepted as a T cell-related cytokine. T helper type 2 (Th2) cells are the major source of IL-13, and it activates tissue fibrosis in a variety of diseases by promoting collagen accumulation in fibroblasts and myofibroblasts and regulating inflammation [10-16].

In regards to the relationship between wound healing and immunity, recent developments in "immunonutrition" may contribute to the enhanced healing of chronic wounds such as pressure ulcers [17-20].

Immunonutrition involves nutritional support through the addition of "immunonutrients" such as amino acids (arginine [Arg] and glutamine [Gln]), omega-3 (n-3) fatty acids vitamins or nucleic acids. These nutrients have been shown to have the immune-modulating (or enhancing) effects via several mechanisms, leading to better outcomes after critical illness or surgical operation. For example, Arg and omega-3 fatty acids are suggested to maintain T cell immunity [21], where as Gln is shown to enhance the release of growth hormone and supports protein synthesis [22].

These observations suggest that, considering the potential clinical effect of immunonutrition (in at least a subset of patients), "immune-regulating" nutrients, in particular the amino acids would have some effect also in mast cells in wound in situ. In this regard, recently, Lechowski, et al. [23] demonstrated that human intestinal mast cells cultured in different culture medium containing different amino acid concentrations (Arg/ 
Gln) showed decreased IgE-mediated release of leukotrienes and cytokines. The authors concluded that the amino acids exert antiinflammatory effect on IgE-dependent mast cell activation. The finding provided in vitro evidence that Arg and Gln would be key nutrients in mast cell-mediated responses. However, it has not been known how these amino acids might modulate mast cell activation when mast cells were activated in an IgE-independent inflammatory stimulation.

In the present in vitro study, we aimed at assessing the potential role of nutrients in mast cell activation by evaluating the effect of the amino acids Gln and Arg in culture milieu on mast cell function with a focus on histamine and IL-13 release.

\section{Materials and Methods}

\section{Cell cultures}

The mouse mastocytoma cell line, P815, was provided by the RIKEN BRC through the National Bio-Resource Project of the MEXT, Japan. The cells were cultured at $37^{\circ} \mathrm{C}$ in an atmosphere of $5 \% \mathrm{CO}_{2}$ in the RPMI-1640 medium (Wako Pure Chemical Industries, Ltd., Osaka, Japan) that was supplemented with $10 \%$ fetal bovine serum (FBS) and 1\% penicillin-streptomycin (P/S). The culture medium contained $300 \mathrm{mg} / \mathrm{L} \mathrm{L}-\mathrm{Gln}$ and $200 \mathrm{mg} / \mathrm{L}$ L-Arg as its basic composition. The culture medium was regularly changed, approximately twice a week, until reaching confluence. Cells were then subjected to further culture for $24 \mathrm{~h}$ in media with different amino acid concentrations. Specifically, the levels of Gln in the medium were adjusted to $500 \mathrm{mg} / \mathrm{L}$ and $1000 \mathrm{mg} / \mathrm{L}$, and the levels of Arg were adjusted to $400 \mathrm{mg} / \mathrm{L}$, in the presence of both $0 \mathrm{mg} / \mathrm{L}$ and $500 \mathrm{mg} / \mathrm{L} \mathrm{Gln}$. The concentrations were achieved using an L-Gln or L-Arg solution (Wako Pure Chemical Industries, Ltd. Osaka, Japan) and non Gln-containing RPMI-1640 (Wako Pure Chemical Industries, Ltd. Osaka, Japan). The other constituents, including FBS and P/S, were not modified.

The cells were subgrouped to be stimulated or not with recombinant murine IL-6 (20 ng/mL) (Peprotech Inc, Rocky Hill, $\mathrm{NJ}$, USA) for $24 \mathrm{~h}$ in each medium, and the culture supernatant was collected for analyses. The IL- 6 stimulation allowed for focus on the response of the mast cells to inflammatory stimulation rather than an allergen challenge. Previous reports indicate that IL-6 stimulation induces histamine release in P815 cells [4]. The cellular viability and proliferation were assayed using microscopic observation, cell counts were analyzed using trypan blue staining (data not shown), and the MTS assay (CellTiter96 Aqueous Non-Radioactive Cell Proliferation Assay ${ }^{\mathrm{TM}}$, Promega Co., Madison, WI, USA) was conducted according to the manufacturer's instructions.

\section{Enzyme-linked immunosorbent assay (ELISA)}

ELISA was used to measure the secreted levels of histamine and IL-13 in the cultured P815 cells. Specifically, after the in vitro culture, supernatant were collected and subjected to the ELISA analysis using commercially available enzyme immunoassay kits (Histamine Enzyme Immunoassay $\mathrm{Kit}^{\mathrm{TM}}$, SPI Bio Inc., Montigny le Bretonneux, France and Mouse IL-13 Quantikine ${ }^{\mathrm{TM}}$, R\&D, Minneapolis, MN, USA) according to the manufacturers' instructions. Each experiment was performed in triplicate.

\section{Statistical analyses}

The data were calculated and analyzed using Student's $t$-tests or a one-way analysis of variance with Tukey's post hoc tests, as appropriate, using the Ekuseru-Toukei $2010^{\mathrm{TM}}$ software (Social Survey Research Information Co., Ltd., Tokyo, Japan). Differences in the amount of histamine and IL-13 that was secreted were compared between the different conditions within the same amino acid concentration and also between the different concentrations of each amino acid. Comparisons were also conducted between the presence and absence of IL- 6 stimulation for each condition. Results with $\mathrm{p}<0.05$ were considered statistically significant.

\section{Results}

\section{Amino acid concentrations affect mast cell proliferation}

The summary of the initial MTS assay used to test the proliferation of P815 mast cells cultured in different amino acid (Arg and Gln) concentrations is provided in Table 1. Depletion of Gln (in the absence of Arg supplementation) decreased the cellular viability and proliferation, whereas the other conditions did not alter these parameters.

\section{Histamine release from mast cells is regulated by IL-6 and Arg but not Gln}

We cultured P815 mast cells in RPMI media with different concentrations of Gln and Arg. The histamine release remained at basal levels under the usual culture condition (i.e., the standard RPMI- 1640 containing $300 \mathrm{mg} / \mathrm{L}$ of Gln and $200 \mathrm{mg} / \mathrm{L}$ of Arg; Figure 1). Variation in only the levels of Gln $(0-1,000 \mathrm{mg} / \mathrm{L})$ in

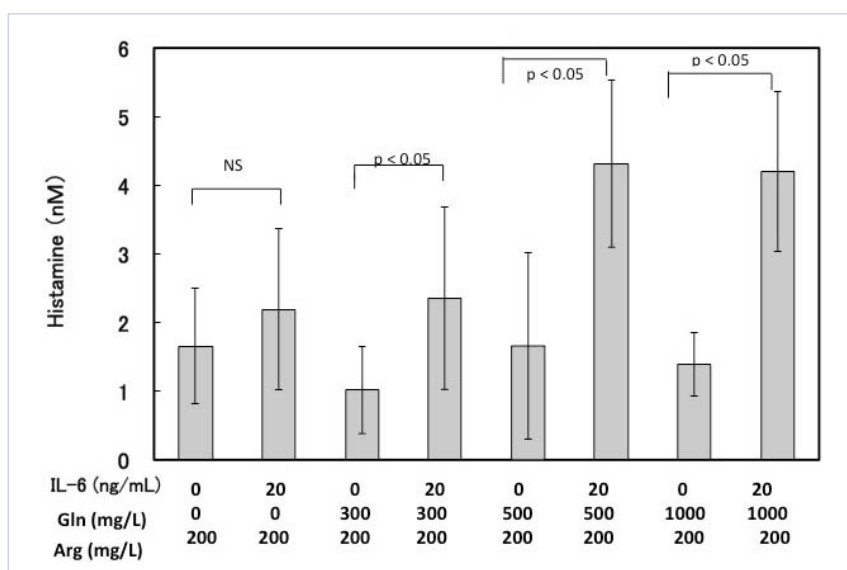

Figure 1: Effect of the glutamine (Gln) concentration on the interleukin (IL)-6-induced histamine release from P815 mast cells.

P815 mast cells were cultured in vitro in RPMI culture media containing different levels of Gln $(0,300,500$ or $1,000 \mathrm{mg} / \mathrm{L})$. The concentrations of histamine in culture supernatants were then measured using enzymelinked immunosorbent assay. Data represent mean values $(n=3)$. 


\begin{tabular}{|c|c|c|c|c|c|c|c|c|}
\hline \multirow{2}{*}{$\frac{\text { Arg }}{(\mathrm{mg} / \mathrm{L})}$} & \multirow{2}{*}{$\frac{\text { Gln }}{(\mathrm{mg} / \mathrm{L})}$} & \multirow{2}{*}{$\begin{array}{c}\text { IL-6 } \\
(\mathrm{ng} / \mathrm{mL})\end{array}$} & \multicolumn{2}{|c|}{ MTS assay } & \multicolumn{2}{|c|}{ Histamine } & \multicolumn{2}{|c|}{ IL-13 } \\
\hline & & & OD & Relative value & $\mathrm{nM}$ & Relative value & $\mathrm{pM}$ & Relative value \\
\hline \multirow{6}{*}{$200 *$} & \multirow{2}{*}{$0^{\#+}$} & 0 & 0.161 & \multirow{2}{*}{0.914} & 2.16 & \multirow{2}{*}{1.214} & 31.50 & \multirow{2}{*}{0.857} \\
\hline & & 20 & 0.147 & & 2.68 & & 27.00 & \\
\hline & \multirow{2}{*}{$300^{\#}$} & 0 & 0.381 & \multirow{2}{*}{0.806} & 1.13 & \multirow{2}{*}{2.317} & 131.39 & \multirow{2}{*}{0.921} \\
\hline & & 20 & 0.307 & & 2.69 & & 121.01 & \\
\hline & \multirow{2}{*}{$500^{\#}$} & 0 & 0.239 & \multirow{2}{*}{1.357} & 1.83 & \multirow{2}{*}{3.280} & 192.72 & \multirow{2}{*}{1.042} \\
\hline & & 20 & 0.324 & & 6.00 & & 200.86 & \\
\hline \multirow{6}{*}{$400 *$} & \multirow{2}{*}{$0^{\#}$} & 0 & 0.242 & \multirow{2}{*}{0.644} & 13.55 & \multirow{2}{*}{1.411} & 21.99 & \multirow{2}{*}{0.838} \\
\hline & & 20 & 0.156 & & 19.11 & & 18.44 & \\
\hline & \multirow{2}{*}{$300^{\#}$} & 0 & 0.391 & \multirow{2}{*}{0.531} & 16.86 & \multirow{2}{*}{1.266} & 106.83 & \multirow{2}{*}{1.004} \\
\hline & & 20 & 0.208 & & 21.35 & & 107.31 & \\
\hline & \multirow{2}{*}{$500^{\#}$} & 0 & 0.312 & \multirow{2}{*}{1.383} & 18.76 & \multirow{2}{*}{1.096} & 173.97 & \multirow{2}{*}{1.064} \\
\hline & & 20 & 0.431 & & 20.56 & & 185.03 & \\
\hline
\end{tabular}

Table 1: Summary of the P815 responses in the presence of varying glutamine (Gln)/arginine (Arg) concentrations and interleukin (IL)-6 stimulation. Results of the MTS assay, histamine enzyme-linked immunosorbent assay (ELISA), and IL-13 ELISA are summarized, and the relative values of the responses to IL-6 (i.e. values of IL-6-stimulated cells/values of non-stimulated cells) are provided.

*,": compared between groups using a one-way analysis of variancewith Tukey's post hoc tests.

Arg = arginine; Gln = glutamine; IL-6 = interleukin-6; IL-13 = interleukin-13

$+:$ cells cultured in the Glu (0) medium had lower viability and the results varied

the RPMI medium did not alter the level of histamine release from the P815 cells $(F(2,22)=0.31, p=0.74)$. On the other hand, P815 cells significantly increased the release of histamine when stimulated with IL- 6 in vitro $(F(1,12)=14.34, p=0.0026)$. The response of the cells to IL-6 differed according to the Gln concentrations in the media with a greater histamine release resulting from IL-6 stimulation in greater Gln concentrations (Figure 1 and Table 1).

Next, we modified the concentrations of both Gln and Arg in the culture media. The summary of the histamine release from the P815 cells in the presence of the different combinations of Arg and Gln concentrations are provided in Figure 2. Compared to those cultured in "normal" Arg (200 mg/L), cells grown in the "high" Arg (400 mg/L) medium released significantly higher levels of histamine $(F(1,11)=24.26, p=0.0005)$, irrespective of the Gln concentrations.

\section{Distinct regulation of IL-13 production from mast cells}

We then explored the effect of the amino acid concentration in the medium on the IL-13 production from the mast cells. Figure 3 and Table 1 illustrate the levels of IL-13 secretion in the presence of IL- 6 and varying concentrations of Gln. IL-13 production was clearly observed in the P815 cells cultured in high concentrations of Gln (500 and 1,000 mg/L), but not in the media that did not contain Gln (Figure 3). In contrast to histamine, the IL-13 level was not significantly changed by in vitro stimulation with IL-6 (Figure 3).

Following this, the production of IL-13 was compared between the different combinations of Arg and Gln concentrations (Figure 4 and Table 1; Figs 3 and Figs 4 represents results from separate experiments). IL-13 production increased in the presence of Gln, regardless of the Arg concentration. More specifically, when the P815 cells were cultured in the RPMI medium with an Arg concentration of $200 \mathrm{mg} / \mathrm{L}$, the IL-13 levels significantly increased with increasing Gln concentrations $(0,300$, and 500 $\mathrm{mg} / \mathrm{L})(\mathrm{F}(2,12)=7.18, \mathrm{p}=0.0089)$.

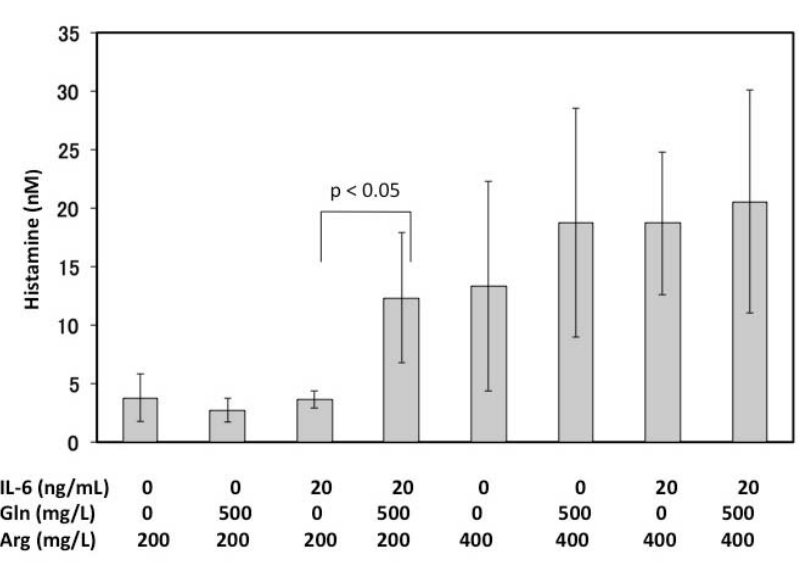

Figure 2: Effect of the combined arginine (Arg) and glutamine (Gln) concentrations in the interleukin (IL)-6-induced histamine release from P815 mast cells.

P815 mast cells were cultured in vitro in RPMI culture media containing different levels of Gln and Arg (as indicated). The concentrations of histamine in culture supernatants were then measured using enzymelinked immunosorbent assay. Data represent mean values $(n=3)$.

\section{Discussion}

Our results demonstrate that the in vitro culture of mast cells with different Gln and Arg levels results in distinct histamine and IL-13 responses, suggesting that amino acid concentrations may play an important role in situ in mast cell activation.

The contribution of amino acids, including Gln and Arg, in "immunonutrition" and wound healing has been widely proposed. For instance, enteral nutrition supplemented with a combination of immunonutrients, including Arg, Gln, omega-3 fatty acids, and nucleotides, has been demonstrated to prevent infectious complications and decrease mortality rates in critically ill and surgical patients compared with standard enteral nutrition [24-26]. In addition, these amino acids have been indicated in the regulation of lymphocyte function [27]; however, their effect on mast cells has remained mostly unclear. 


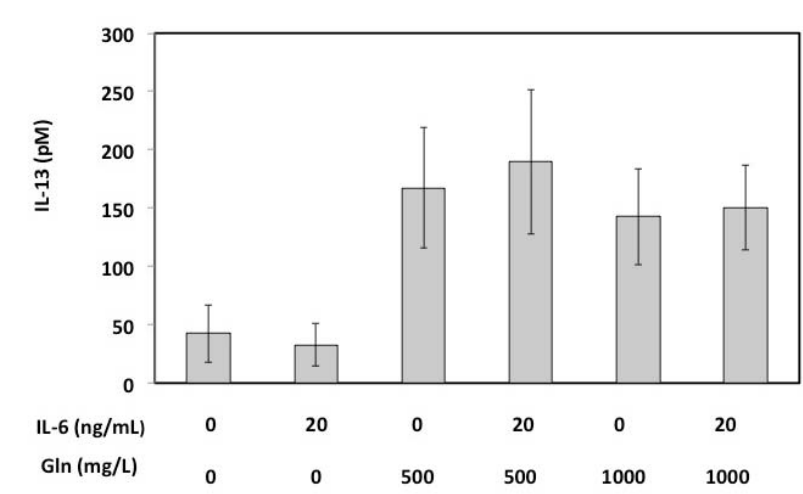

Figure 3: Effect of the glutamine (Gln) concentration on the interleukin (IL)-6-induced IL-13 secretion from P815 mast cells.

P815 mast cells were cultured in vitro in RPMI culture media containing different levels of Gln $(0,300,500$ or $1,000 \mathrm{mg} / \mathrm{L})$. The concentrations of IL-13 in culture supernatants were then measured using enzymelinked immunosorbent assay. Data represent mean values $(n=3)$.

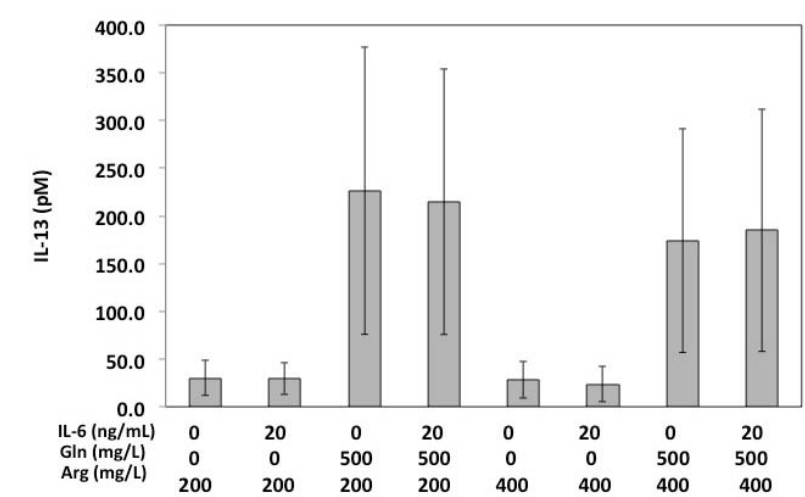

Figure 4: Effect of the combined arginine (Arg) and glutamine (Gln) concentrations on the interleukin (IL)-6-induced IL-13 production from P815 mast cells.

P815 mast cells were cultured in vitro in RPMI culture media containing different levels of Gln and Arg (as indicated). The concentrations of IL13 in culture supernatants were then measured using enzyme-linked immunosorbent assay. Data represent mean values $(\mathrm{n}=3)$.

Our present results demonstrate that Gln and Arg may modulate mast cell functions such as degranulation and IL-13 production. Previous studies have indicated that histamine may stimulate fibroblast migration, implicating the role of histamine in wound healing [8-9]. In addition, IL-13 induces collagen synthesis via induction of transforming growth factor- $\beta$ expression $[12,28]$. Recent studies have reported the role of IL-13/IL-13R signaling in allergic conditions [29], pulmonary fibrosis [30], and gastroenteritis [31,32]. The pro-fibrotic property of IL-13 also plays a role in fibrosis and remodeling (inflammation and construction of vasculature) of the skin as well as wound healing $[33,34]$. Considering this, it is likely that amino acids regulate skin lesions, at least in part, by controlling the levels of mediators that are produced from mast cells, such as histamine and IL-13.

Although we did not investigate the role of omega-3 fatty acids, Park et al. [35] demonstrated that these fatty acids also modulate the Th2 cytokine expression from mast cell lines by regulating the pathways of the transcription factors GATA-1 and/ or GATA-2. The authors did not find a significant change in IL-4 and IL-13 expression from the P815 mast cells because the cell line lacked GATA-1 expression; however, their results suggested that the oral administration of diet-derived nutrients (e.g. omega-3 fatty acids in fish oil) might regulate mast cell function in vivo [35]. Therefore, it would be interesting to investigate the interaction or cumulative effects of fatty acids and amino acids on mast cell function.

In the present study, we set the concentration of L-Gln in the RPMI culture medium as $0 \mathrm{mg} / \mathrm{L}, 300 \mathrm{mg} / \mathrm{L}(2.05 \mathrm{mM}$; the basic composition of RPMI), $500 \mathrm{mg} / \mathrm{L}$ (3.42 mM), and 1,000 $\mathrm{mg} / \mathrm{L}$ (6.84 mM). As for L-Arg, because Arg-free RPMI was not available, we used concentrations of $200 \mathrm{mg} / \mathrm{L}(1.15 \mathrm{mM}$; the basic composition), and $400 \mathrm{mg} / \mathrm{L}$ (2.3 mM) by addition of L-Arg to the original medium. These concentrations were higher than the physiological concentrations, as according to Lechowski et al. [23] the physiological serum concentrations of the amino acids are reported to be $0.6 \mathrm{mM}$ for L-Gln and $0.1 \mathrm{mM}$ for L-Arg. Thus our results here may reflect only in vitro findings using cultured cell line, however, further in vivo studies e.g. by using oral feeding of the immunonutrients to experimental animals would enhance understanding of the mechanism of immunonutrition in mast cell responses. Further, it should be tested another experimental conditions (e.g. different doses of amino acids and cytokine, different culture period, etc), and using another mast cell line including humans, since P815 murine mastocytoma cell line is reported to lack FCER expression [36] and thus would not reflect the nature of mature human mast cells in vivo.

In conclusion, we found that amino acids may play a role in the regulation of mast cell activation in the presence of inflammatory stimulation. Clarification of the role of amino acids and other nutrients in the function of mast cells may provide new avenues for establishing novel therapeutic strategies for a number of inflammatory conditions.

\section{Conclusions}

The release of histamine and expression of IL-13 by mast cells in response to inflammatory stimulation may be altered, at least in part, by amino acid concentrations in vitro. Regulating amino acids levels may therefore be important for the modification of mast cell function during inflammation as well as in the healing process of chronic wounds.

\section{Acknowledgements}

The authors thank Ms. Yuka Shimura and Ms. Toshiko Mogi for their excellent technical assistance and Ms. Ami Hirota for secretarial support. This work was supported by JSPS KAKENHI Grant Number 23617028.

\section{References}


1. Piliponsky AM, Gleich GJ, Nagler A, Bar I, Levi-Schaffer F (2003) NonIgE-dependent activation of human lung- and cord blood-derived mast cells is induced by eosinophil major basic protein and modulated by the membrane form of stem cell factor. Blood 101(5): 1898-1904.

2. Okayama Y, Saito H, Ra C (2008) Targeting human mast cells expressing G-protein-coupled receptors in allergic diseases. AllergologyInt 57(3):197-203.

3. Saito H, Ishizaka T, Ishizaka K (2013) Mast cells and IgE: from history to today. Allergology Int 62(1): 3-12.

4. Zhang H, Lin L, Yang H, Zhang Z, Yang X, et al. (2010) Induction of IL-13 production and upregulation of gene expression of protease activated receptors in P815 cells by IL-6. Cytokine 50(2):138-145.

5. Trautmann A, Toksoy A, Engelhardt E, Brocker EB, Gillitzer R (2000) Mast cell involvement in normal human skin wound healing: expression of monocyte chemoattractant protein-1 is correlated with recruitment of mast cells which synthesize interleukin-4 in vivo. J Pathol 190(1): 100-106.

6. Abe M, Yokoyama Y, Amano H, Matsushima Y, Kan C, et al. (2002) Effect of activated human mast cells and mast cell-derived mediators on proliferation, type I collagen production and glycosaminoglycans synthesis by human dermal fibroblasts. Eur J Dermatology 12(4): 340-346.

7. Chisholm C, Greene JF Jr (2011) Progression from atypical/dysplastic intraepidermal proliferations and carcinoma in situ to invasive tumors: a pathway based on current knowledge. Am J Dermatopathol 33(8): 803-810.

8. Weller K, Foitzik K, Paus R, Syska W, Maurer M (2006) Mast cells are required for normal healing of skin wounds in mice. FASEB J 20(13): 2366-2368.

9. Kohyama T, Yamauchi Y, Takizawa H, Kamitani S, Kawasaki S, et al (2010) Histamine stimulates human lung fibroblast migration. Mol Cell Biochem 337(1-2): 77-81.

10. Chiaramonte MG, Donaldson DD, Cheever AW, Wynn TA (1999) An IL-13 inhibitor blocks the development of hepatic fibrosis during a T-helper type 2-dominated inflammatory response. J Clin Invest 104(6): 777-785.

11. Kaviratne M, Hesse M, Leusink M, Cheever AW, Davies SJ, et al. (2004) IL-13 activates a mechanism of tissue fibrosis that is completely TGFbeta independent. J Immunol 173(6): 4020-4029.

12. Fichtner-Feigl S, Strober W, Kawakami K, Puri RK, Kitani A (2006) IL13 signaling through the IL-13alpha2 receptor is involved in induction of TGF-beta1 production and fibrosis. Nat Med 12(1): 99-106.

13. Fichtner-Feigl S, Young CA, Kitani A, Geissler EK, Schlitt HJ, et al. (2008) IL-13 signaling via IL-13R alpha2 induces major downstream fibrogenic factors mediating fibrosis in chronic TNBS colitis. Gastroenterology 135(6): 2003-2013.

14. Borowski A, Kuepper M, Horn U, Knupfer U, Zissel G, et al. (2008) Interleukin-13 acts as an apoptotic effector on lung epithelial cells and induces pro-fibrotic gene expression in lung fibroblasts. Clin Exp Allergy 38(4): 619-628.

15. Barron L, Wynn TA (2011) Fibrosis is regulated by Th2 and Th17 responses and by dynamic interactions between fibroblasts and macrophages. Am J Physiol Gastrointest Liver Physiol 300(5): G723G728.

16. Oh MH, Oh SY, Yu J, Myers AC, Leonard WJ, et al. (2011) IL-13 induces skin fibrosis in atopic dermatitis by thymic stromal lymphopoietin. J
Immunol 186(12): 7232-7242.

17. Heyman H, Van De Looverbosch DE, Meijer EP, Schols JM (2008) Benefits of an oral nutritional supplement on pressure ulcer healing in long-term care residents. J Wound Care 17(11): 476-480.

18. Schols JM, Heyman H, Meijer EP (2009) Nutritional support in the treatment and prevention of pressure ulcers: an overview of studies with an arginine enriched oral nutritional supplement. J Tissue Viability 18(3): 72-79.

19. Brewer S, Desneves K, Pearce L, Mills K, Dunn L, et al. (2010) Effect of an arginine-containing nutritional supplement on pressure ulcer healing in community spinal patients. J Wound Care 19(7): 311-316.

20. Yatabe J, Saito F, Ishida I, Sato A, Hoshi M, et al. (2011) Lower plasma arginine in enteral tube-fed patients with pressure ulcer and improved pressure ulcer healing after arginine supplementation by Arginaid Water. J Nutr Health Aging 15(4): 282-286.

21. Okamoto Y, Okano K, Izuishi K, Usuki H, Wakabayashi H, et al. (2009) Attenuation of the systemic inflammatory response and infectious complications after gastrectomy with preoperative oral arginine and omega-3 fatty acids supplemented immunonutrition. World J Surg 33(9): 1815-1821.

22. Welbourne TC (1995) Increased plasma bicarbonate and growth hormone after an oral glutamine load. Am J Clin Nutr 61(5): 1058-1061.

23.Lechowski S, Feilhauer K, Staib L, Coeffier M, Bischoff SC, et al. (2013) Combined arginine and glutamine decrease release of de novo synthesized leukotrienes and expression of proinflammatory cytokines in activated human intestinal mast cells. Eur J Nutr 52(2): 505-512.

24. Suchner U, Kuhn KS, Furst P (2000) The scientific basis of immunonutrition. Proc Nut Soc 59(4): 553-563.

25. Heyland DK, Novak F, Drover JW, Jain M, Su X, et al. (2001) Should immunonutrition become routine in critically ill patients? A systematic review of the evidence. JAMA 286 (8): 944-953.

26. Kim H (2011) Glutamine as an immunonutrient. Yonsei Med J 52(6): 892-897.

27. Grimble RF (2001) Nutritional modulation of immune function. Proc Nutr Soc 60(3): 389-397.

28. Wynn TA (2008) Cellular and molecular mechanisms of fibrosis. J Pathol 214(2): 199-210.

29. Plante S, Semlali A, Joubert P, Bissonnette E, Laviolette M, et al. (2006) Mast cells regulate procollagen I (alpha 1) production by bronchial fibroblasts derived from subjects with asthma through IL-4/IL-4 delta 2 ratio. J Allergy Clin Immunol 117(6): 1321-1327.

30.Wilson MS, Wynn TA (2009) Pulmonary fibrosis: pathogenesis, etiology and regulation. Mucosal Immunol 2(2): 103-121.

31. Bailey JR, Bland PW, Tarlton JF, Peters I, Moorghen M, et al. (2012) IL-13 promotes collagen accumulation in Crohn's disease fibrosis by down-regulation of fibroblast MMP synthesis: a role for innate lymphoid cells? PloS One 7(12): e52332.

32. Souza AL, Souza PR, Pereira CA, Fernandes A, Guabiraba R, et al. (2011) Experimental infection with Schistosoma mansoni in CCR5deficient mice is associated with increased disease severity, as CCR5 plays a role in controlling granulomatous inflammation. Infect Immun 79(4): 1741-1749. 
33. Zheng T, Oh MH, Oh SY, Schroeder JT, Glick AB, et al. (2009) Transgenic expression of interleukin-13 in the skin induces a pruritic dermatitis and skin remodeling. J Invest Dermatol 129(3): 742-751.

34. Gause WC, Wynn TA, Allen JE (2013) Type 2 immunity and wound healing: evolutionary refinement of adaptive immunity by helminths. Nat Rev Immunol 13: 607-614.
35. Park BK, Park S, Park JB, ParkMC, Min TS, et al. (2013) Omega-3 fatty acids suppress Th2-associated cytokine gene expressions and GATA transcription factors in mast cells. J Nutr Biochem 24(5): 868-876.

36. Thathiah P, Sanapala S, Rodriguez AR, Yu JJ, Murthy AK, et al. (2011) Non-FceR bearing mast cells secrete sufficient interleukin-4 to control Francisella tularensis replication within macrophages. Cytokine 55(2): 211-20. 